\title{
Comparisons Based on Event-related Potentials in Dental Students with Different Levels of Experience Knowledge
}

\author{
Kenji Doi, ${ }^{1}$ Tomoyasu Ebihara, ${ }^{2}$ and Shinichiro Aoki ${ }^{2,3}$ \\ ${ }^{1}$ Nihon University Graduate School of Dentistry at Matsudo, Oral Diagnosis, Matsudo, Chiba 271-8587, Japan \\ ${ }^{2}$ Department of Oral Diagnosis, Nihon University School of Dentistry at Matsudo, Matsudo, Chiba 271-8587, Japan, ${ }^{3}$ Research \\ Institute of Oral Science, Nihon University School of Dentistry at Matsudo, Matsudo, Chiba 271-8587, Japan
}

\section{Article History}

Received 28 November 2016

Accepted 9 December 2016

\section{Keywords :}

pattern cognition,

experience knowledge,

tooth type identification,

mental rotation

\begin{abstract}
The information handling processes for character and tooth recognition tasks were compared between the dental students who had acquired different knowledge levels. The subjects included 20 second-year students who had acquired textbook-based knowledge and 20 fifth-year students who had acquired both textbook-based and experience knowledge though ongoing clinical exercise. The task was to differentiate diagrams depicting a "katakana character" or a "tooth." The diagrams were randomly presented at a ratio of 2: 8 according to the oddball paradigm. The subjects were instructed to push a button only when a target stimulus was presented. Average waveforms were determined by averaging ERP waveforms derived at $\mathrm{Pz}$ for a principal component analysis (PCA).

The results showed the same trends in information for character diagrams in both year students; the contribution rates of the PCA component representing pattern matching or attention-orientation as well as working memory update was higher. Similar trends were observed in information for tooth diagrams in terms of a greater percentage of the PCA components represented cognition and decision and behavior performance, but in the fifthyear students P3a that represented attention-orientation was extracted independently.

These results indicate similar information in both-year students for the character task, whereas fifth-year students who had acquired experience knowledge appeared to make their decisions based on more effective thinking for the tooth task.
\end{abstract}

\section{Introduction}

Dentists in their routine clinical practice typically employ an intuitive diagnostic process called pattern cognition when making clinical diagnoses (1-3). We would expect highlyqualified dentists who understand distinctive disease patterns to make more efficient diagnoses. High-level visual cognition in the brain may involve hierarchical processing of morphological information of an object in the pathway from the occipital to the temporal lobe of the cerebrum. Cells that respond to specific given line or edge patterns have been identified in the primary visual area where information is initially received and processed, but the detailed mechanism of high-level tasks, such as pattern cognition and thinking,

Correspondence to :

Shinichiro Aoki

E-mail : aoki.shinichiro@nihon-u.ac.jp remains to be elucidated from the standpoint of cognitive psychology in the brain.

We have been reported the pattern cognition, in the tooth type identification (4-6). To elucidate the pattern cognition whereby tooth patterns in the brain are immediately matched to tooth morphology and profiles presented before the eyes, a study of brain activity is beneficial. For this purpose, event-related potentials (ERP) (7-10), which are provoked by psychological tasks including cognition, discrimination, and task solution, have been commonly employed.

The ERP provided by task solution can guess a psychology action by analyzing various wave pattern ingredients. The purpose of the present study was to elucidate the effects of clinical experience knowledge on information processing in the levels of sophistication 


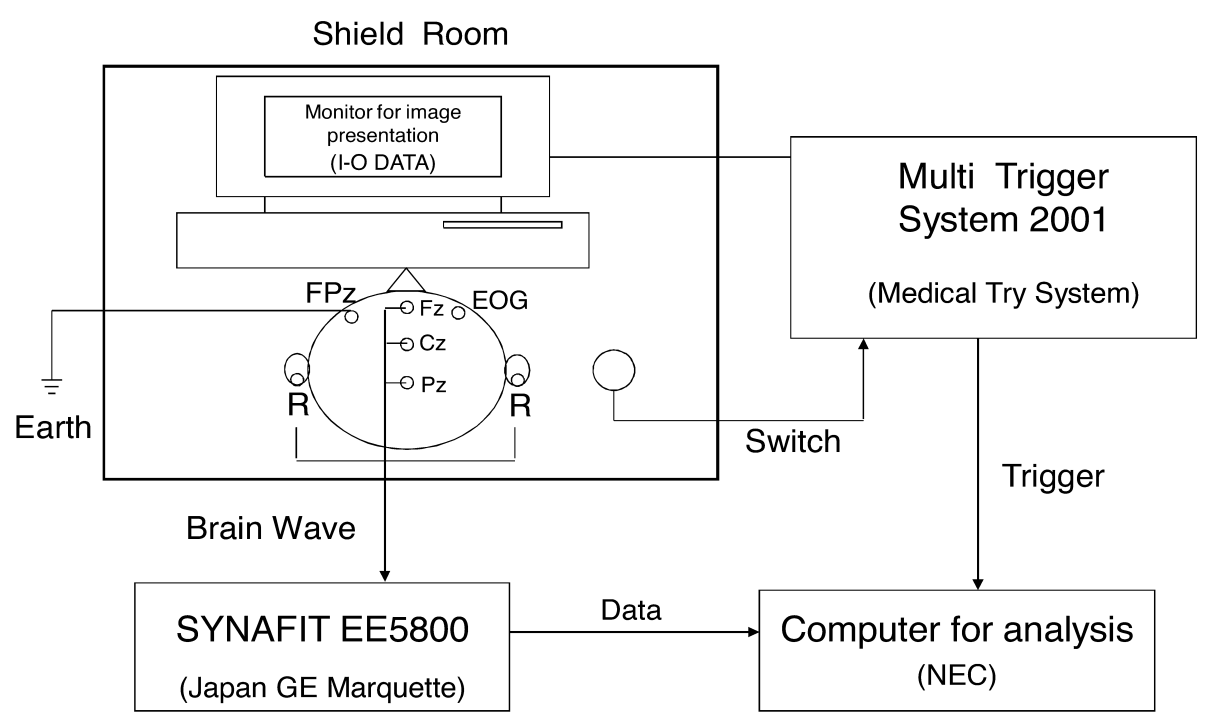

Fig. 1. ERP measurement/analysis system

achieved by dental students within a cognitive psychology framework by comparing trends in the occurrence of certain ERP waveform components $(11,12)$.

\section{Materials and Methods}

Subjects consisted of 20 second-year students (mean age: 19.3 years, $\mathrm{SD} \pm 1.2$ years) who had acquired dental anatomical knowledge by the lecture and exercise including sketch and curving of the each tooth form, and fifth-year students (mean age: 23.0 years, $\mathrm{SD} \pm 1.3$ years) who had acquired both anatomical knowledge in textbook and some clinical experience knowledge in ongoing clinical exercise after going through basic simulation laboratory study. All subjects were right-handed, had no history of psychiatric disease, and had no visual impairments that would affect the results of our studies.

The dental students have been learned liberal arts and some subjects related to the dental science including dental anatomy (including the practice of sketch and carving of the tooth) in the first year, and fundamental medical and dental sciences including anatomy, physiology, biochemistry and so on in the second year. They have been learned fundamental clinical exercise including dental prosthesis and retention in the third and fourth years. The fifth-year students had attended lectures and had been exposed to clinical exercise (including preparation of tooth crown and bridge), and their knowledge related to the tooth morphology is more improved than in the second year.

Measurements were performed in a shielded room by the method of Kuwahara and Ebihara (13). The subjects sat on a chair at rest with their head fixed. Images were presented on a monitor positioned $50 \mathrm{~cm}$ in front of the subject. Electrooculograms (EOGs) representing indicators of brain waves and artifacts were obtained after presenting task images on the monitor. Brain waves were recorded using a SYNAFIT EE5800 (NEC Medical Systems). ERP waveforms were extracted from the brain waves for analysis.

Fig.1 shows the ERP measurement/analysis system. The sampling frequency for the brain waves was $500 \mathrm{~Hz}$. Brain waves were derived at three locations, $\mathrm{F} z, \mathrm{C} z$, and $\mathrm{Pz}$, in bilateral earlobes based on the 10-20 system. Silver/silver chloride electrodes were used, with a contact impedance of 5 $\mathrm{k} \Omega$ or lower. Low- and high-frequency filters were set to 0.1 and $100 \mathrm{~Hz}$, respectively. Diagrams of characters and teeth were used as images $(480 \times 480$ pixels). The images used for individual tasks are described below.

\section{Character task}

One target stimulus was a katakana character printed with the normal orientation of “ア." Frequent stimuli included the normally oriented character "マ" and left-toright reversed versions of the characters “ア” and “マ." The katakana characters consist merely of simple points and lines, and can be easily distinguished. We selected characters with the same number of strokes and similar shapes for the target and frequent stimuli. The images rotated at angles of 90,180 and 270 degrees clockwise assuming that a normally oriented image was defined as $0^{\circ}$ 
Target stimulus (normally oriented katakana character " $\boldsymbol{~}$ ")

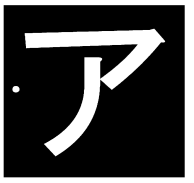

$0^{\circ}$

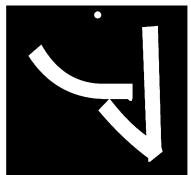

$90^{\circ}$

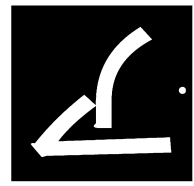

$180^{\circ}$

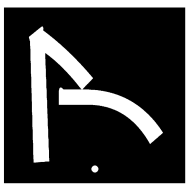

$270^{\circ}$

Frequent stimulus (normally oriented katakana character "マ")

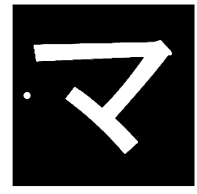

$0^{\circ}$

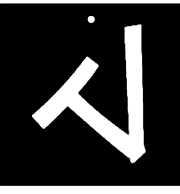

$90^{\circ}$

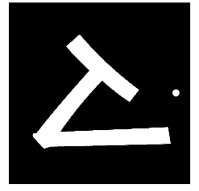

$180^{\circ}$

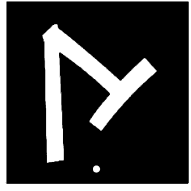

$270^{\circ}$

Frequent stimulus (left-to-right reversed version of the katakana character " $\boldsymbol{P}$ ")

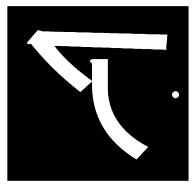

$0^{\circ}$

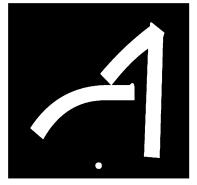

$90^{\circ}$

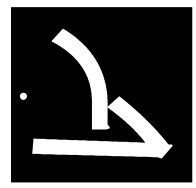

$180^{\circ}$

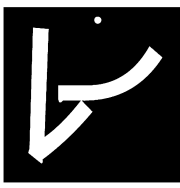

$270^{\circ}$

Frequent stimulus (left-to-right reversed version of the katakana character "マ")

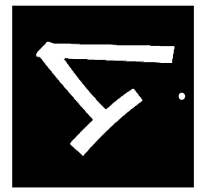

$0^{\circ}$

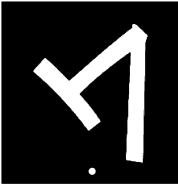

$90^{\circ}$

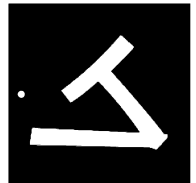

$180^{\circ}$

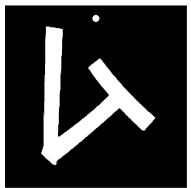

$270^{\circ}$

Fig. 2. Diagrams used for the katakana character task

We used images turned 90,180 , and $270^{\circ}$ clockwise, with normal orientation defined as $0^{\circ}$.

Target stimulus (right mandibular 1st molar)

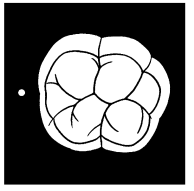

$0^{\circ}$

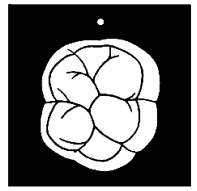

$90^{\circ}$

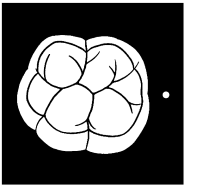

$180^{\circ}$

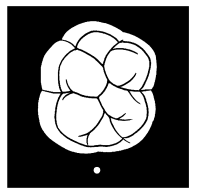

$270^{\circ}$

Frequent stimulus (left maxillary 1st molar)

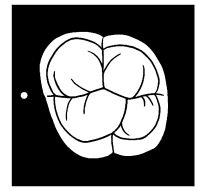

$0^{\circ}$

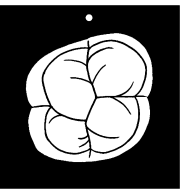

$90^{\circ}$

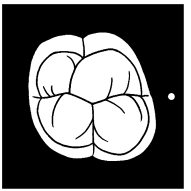

$180^{\circ}$

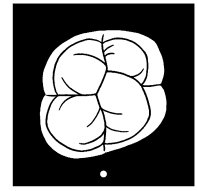

$270^{\circ}$
Frequent stimulus (left mandibular 1st molar)

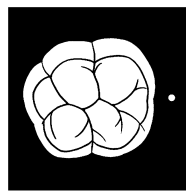

$0^{\circ}$

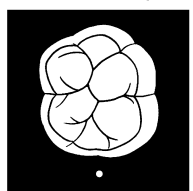

$90^{\circ}$

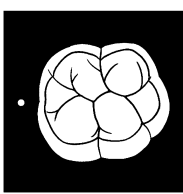

$180^{\circ}$

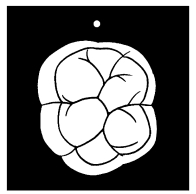

$270^{\circ}$

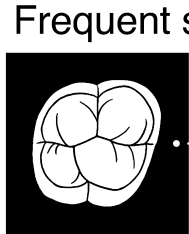

$0^{\circ}$

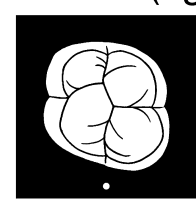

$90^{\circ}$

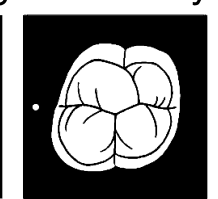

$180^{\circ}$

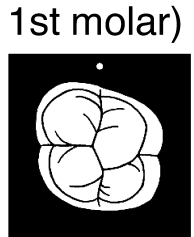

$270^{\circ}$

Fig. 3. Diagrams used for the tooth task

We used diagrams turned 90,180 , and $270^{\circ}$ clockwise, with upward orientation of the buccal side defined as $0^{\circ}$.

were used Fig.2.

\section{Tooth task}

The target stimulus was the right mandibular 1st molar. The frequent stimuli were the left maxillary 1st molar, the left mandibular 1st molar, and the right maxillary 1st molar. We used diagrams rotated at $90^{\circ}$ in the same manner as the character task, defining as $0^{\circ}$ as in the condition in which the buccal side is oriented upward Fig.3. To prevent decision- making while remembering some shapes, a mark was applied to the mesial position of the diagrams. The subjects were instructed to confirm the orientation of the shape before decision-making.

\section{ERP recording/analysis}

The images were randomly presented one after another according to the oddball paradigm using a Multi Trigger System 2001. The target and frequent stimuli were 
Table 1. Meaning of the cognitive psychology of the ERP waveforms

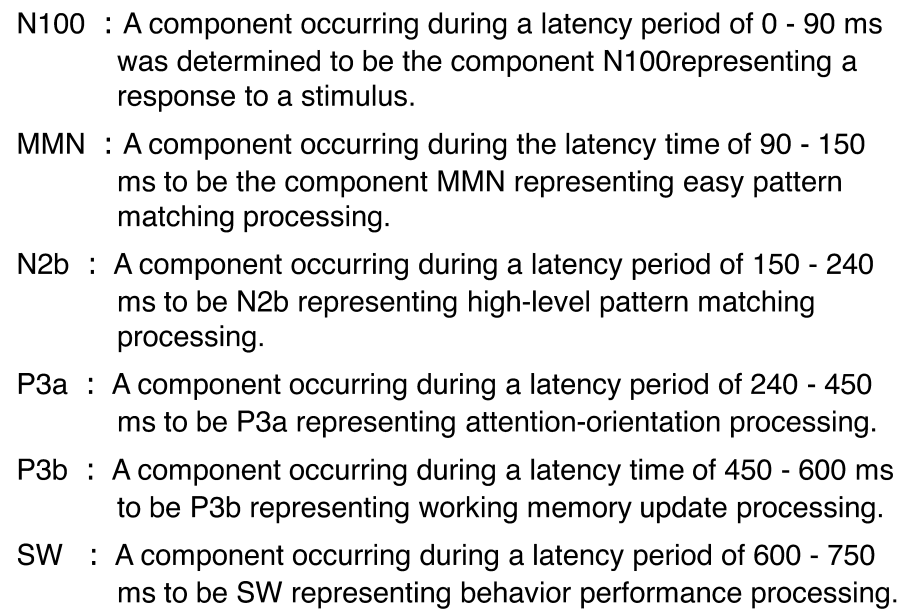

presented at a ratio of 2: 8 . Each image was presented for $1500 \mathrm{~ms}$, and each task was presented 500 times. The subjects were instructed to push a button only when the target stimulus was presented. The correct answers were 24 in total. The subjects who could get less than 20 correct answers were excluded. An average waveform, from which noises and arch facts are removed, was created by adding up 20 ERP wave patterns that each subject provided when target stimulus were presented. All average waveforms from each subject were added up to create a general average waveform. Then, the subjects' average waveforms were sorted out according to angles at which target stimulus were rotated, and added up in each angle category to create angle specific average waveforms. Then, from the subjects' average waveforms, angle-specific average waveforms were generated for each subject. Commonly observed waveforms of $\mathrm{Pz}$ were used because they were likely to demonstrate the profiles of each task at $0^{\circ}$.

To investigate information processing primarily for cognitive performance, we averaged waveform components with a latency period of 0-750 $\mathrm{ms}$ for the average waveforms over $30 \mathrm{~ms}$, followed by a principal component analysis for 25-point waveform components. We used statistical software (PASW Stastics18, SPSS Inc.) for statistical processing. The identification of the latency guessed it in reference to eigenvalue indicating the information of each principal component, a value of the factor loading. In principal component analysis, we extracted ERP waveform components if they had a characteristic value of 2.0 or above and a factor loading of 0.7 or above.

An ERP waveform component was identified by the following criteria: a component occurring during a latency period of 0-90 ms was determined to be the component N100, representing a response to a stimulus; a component occurring during the latency time of $90-150 \mathrm{~ms}$ to be the component MMN, representing easy pattern matching processing; a component occurring during a latency period of 150-240 ms to be N2b, representing high-level pattern matching processing; a component occurring during a latency period of $240-450 \mathrm{~ms}$ to be $\mathrm{P} 3 \mathrm{a}$, representing attention-orientation processing; a component occurring during a latency time of 450-600 ms to be P3b, representing working memory update processing; and a component occurring during a latency period of 600-750 ms to be SW, representing behavior performance processing Table 1 (14-17). To avoid loads imposed by the sequence of images presented, the tasks were conducted in a shifted sequence in each subject. The duration from preparation to completion of the experiments was about 90 minutes, with a resting time of 10 minutes between tasks.

The present study was approved by from the Ethical Committee of the Nihon University School of Dentistry at Matsudo for the experiments (approval number: EC16-1511-010-2) and, prior to this study, we explained to the subjects the purpose of the experiments, and obtained their informed consent.

\section{Results}

\section{Reaction time of the tasks}

As for reaction times, both second and fifth -year students take longer to perform the tooth task than the character task; $698 \pm 185$ and $467 \pm 83 \mathrm{~ms}$, and $663 \pm 185$ and $475 \pm 83$ 
Table 2. Comparison of reaction times, VAS and correct answer rates

\begin{tabular}{|c|c|c|c|c|}
\hline & & $\begin{array}{l}\text { Reaction times } \\
\quad(\mathrm{ms})\end{array}$ & VAS & $\begin{array}{l}\text { Correct answer } \\
\text { rates }(\%)\end{array}$ \\
\hline \multirow[t]{2}{*}{$\begin{array}{l}\text { Second-year } \\
\text { students }\end{array}$} & character task & $\begin{array}{l}534 \\
(67)\end{array}$ & ${ }^{29}{ }^{(15)}$ & $\begin{array}{c}100 \\
(1)\end{array}$ \\
\hline & tooth task & $\begin{array}{c}698 \text { لـ } \\
(134)\end{array}$ & $\begin{array}{l}63\rfloor \\
(26)\end{array}$ & $\begin{array}{l}98 \\
(5)\end{array}$ \\
\hline \multirow[t]{2}{*}{$\begin{array}{l}\text { Fifth-year } \\
\text { students }\end{array}$} & character task & $\begin{array}{l}475 \\
(83)\end{array}$ & $\begin{array}{c}26 \\
(15)\end{array}$ & $\begin{array}{c}100 \\
(0)\end{array}$ \\
\hline & tooth task & $\begin{array}{c}663-ل \\
(185)\end{array}$ & $\begin{array}{c}53 \\
(25)\end{array}$ & $\begin{array}{l}98 \\
(4)\end{array}$ \\
\hline
\end{tabular}

ms, respectively. No significant differences were observed between the second and fifth year students (Table 2).

\section{Subjective difficulty of the tasks (VAS)}

In second-year students, the VAS score was significantly higher for the tooth task than for the character task $(63 \pm 26$ and $29 \pm 15$, respectively). In fifth-year students, scores were significantly higher for the tooth task than for the character task ( $53 \pm 25$ and $26 \pm 15$, respectively). No significant differences were observed between the second and fifth year students (Table 2).

\section{Correct answer rates for the tasks}

In second-year students, no significant differences were observed in the rate between the character and tooth tasks (98 \pm 5 and $100 \pm 1 \%$, respectively) (Table 2). In fifth-year students, no significant differences were observed in the rate between the character and tooth tasks $(100 \pm 0$ and $98 \pm$ $4 \%$, respectively). No task-specific difference was observed between the second and fifth year students (Table 2).

Trends in extraction of ERP waveform components for the character task

As for the results of the principal component analysis for the character task, in second-year students, we found that the primary principal component for the character task was a composite of $\mathrm{N} 2 \mathrm{~b}$ and $\mathrm{P} 3 \mathrm{a}$ (Table 3 ), with a characteristic value of 6.42 ; the secondary principal component was P3b, with a value of 5.72 ; the tertiary principal component was SW, with a value of 3.99; and the quaternary principal component was MMN, with a value of 2.70. The cumulative proportion for the primary to quaternary principal components was $75.3 \%$.

In fifth-year students, we found that the primary principal component for the character task was a composite of $\mathrm{N} 2 \mathrm{~b}$ and P3a, with a characteristic value of 7.26; the secondary principal component was P3b (Table 3 ), with a value of 5.58 ; the tertiary principal component was SW, with a value of 3.51; and the quaternary principal component was MMN, with a value of 3.19. The cumulative proportion for the primary to quaternary principal components was $78.2 \%$.

Trends in extraction of ERP waveform components for the tooth task

As for the results of the principal component analysis for the tooth task, in the second and the fifth-year students, cumulative proportion from first to fourth principal components (characteristic value of 2.0 or above) were more than 70\% (Table 4). When investigating the trend in ERP components extracted for the tooth task in second-year students, we found the primary principal component of the tooth task was a composite of P3a and P3b, with a characteristic value of 9.41 ; the secondary principal component was SW, with a value of 5.62; and the tertiary principal component was a composite of $\mathrm{MMN}$ and $\mathrm{N} 2 \mathrm{~b}$, with a value of 3.53 . The cumulative proportion of primary to tertiary principal components was $74.2 \%$.

When investigating the trend in ERP components extracted for the tooth task in fifth-year students, we found that the primary principal component of the tooth task was 
Table 3. Trends in ERP components extracted for information processing for characters

\begin{tabular}{|c|c|c|c|c|c|c|c|c|c|}
\hline \multicolumn{6}{|c|}{ Second year } & \multicolumn{4}{|c|}{ Fifth year } \\
\hline Latency & PC1 & $\mathrm{PC} 2$ & PC3 & $\mathrm{PC} 4$ & ERP components & $\mathrm{PC1}$ & $\mathrm{PC} 2$ & PC3 & $\mathrm{PC} 4$ \\
\hline $0-30$ & 0.146 & 0.254 & 0.130 & 0.590 & & -.005 & .167 & -.129 & -.147 \\
\hline $31-60$ & 0.229 & 0.225 & 0.414 & 0.508 & N100 & .199 & .119 & .066 & .204 \\
\hline $61-90$ & -0.102 & -0.014 & -0.344 & 0.631 & & 204 & 131 & .188 & .778 \\
\hline $91-120$ & -0.230 & 0.089 & -0.179 & 0.887 & & .019 & .335 & .485 & .710 \\
\hline $121-150$ & 0.294 & 0.046 & 0.281 & 0.721 & IVIIVIIN & .162 & 296 & -.007 & .726 \\
\hline $151-180$ & 0.827 & 0.144 & 0.148 & 0.032 & & 417 & -.034 & -.028 & .678 \\
\hline $181-210$ & 0.768 & 0.364 & -0.073 & 0.208 & N2b & .466 & -.158 & .236 & .675 \\
\hline $211-240$ & 0.713 & 0.625 & -0.021 & 0.366 & & 727 & -.055 & 269 & 446 \\
\hline $241-270$ & 0.824 & 0.355 & 0.083 & 0.015 & & .796 & .108 & .192 & .376 \\
\hline $271-300$ & 0.742 & 0.481 & 0.299 & 0.017 & & .888 & .183 & .079 & .361 \\
\hline $301-330$ & 0.787 & 0.369 & 0.285 & 0.082 & & .928 & .158 & .067 & .208 \\
\hline $331-360$ & 0.898 & 0.191 & 0.222 & 0.001 & P3a & .916 & .072 & .151 & .104 \\
\hline $361-390$ & 0.901 & 0.259 & 0.168 & -0.007 & & .895 & .108 & .208 & .094 \\
\hline $391-420$ & 0.835 & 0.268 & 0.262 & 0.072 & & .859 & .241 & .217 & -.008 \\
\hline $421-450$ & 0.767 & 0.255 & 0.401 & 0.042 & & 806 & 394 & .116 & -.027 \\
\hline $451-480$ & 0.587 & 0.492 & 0.257 & 0.052 & & .705 & .580 & .108 & .004 \\
\hline $481-510$ & 0.418 & 0.715 & 0.150 & 0.008 & & .348 & .842 & .205 & .022 \\
\hline $511-540$ & 0.251 & 0.817 & 0.248 & 0.066 & P3b & .130 & .945 & .165 & -.044 \\
\hline $541-570$ & 0.179 & 0.836 & 0.357 & 0.139 & & .183 & .907 & .132 & .085 \\
\hline $571-600$ & 0.167 & 0.782 & 0.428 & 0.209 & & .108 & 921 & .188 & .159 \\
\hline $601-630$ & 0.206 & 0.671 & 0.450 & 0.326 & & .047 & .803 & .397 & .219 \\
\hline $631-660$ & 0.169 & 0.125 & 0.810 & 0.099 & & .191 & .651 & .591 & .215 \\
\hline $661-690$ & 0.242 & 0.347 & 0.859 & 0.072 & SW & .232 & .435 & .839 & .124 \\
\hline $691-720$ & 0.267 & 0.260 & 0.892 & 0.038 & & .214 & .295 & .899 & .119 \\
\hline $721-750$ & 0.284 & 0.242 & 0.886 & -0.072 & & .320 & 190 & 873 & .134 \\
\hline $\begin{array}{l}\text { character } \\
\text { value }\end{array}$ & 6.42 & 5.72 & 3.99 & 2.70 & & 7.26 & 5.58 & 3.51 & 3.19 \\
\hline $\begin{array}{l}\text { Cumlautive } \\
\text { proportion }\end{array}$ & 25.7 & 48.6 & 64.5 & 75.3 & & 29.0 & 51.3 & 65.4 & 78.2 \\
\hline
\end{tabular}

principal component. Cumulative proportion shows ratio that each principal component accounts for in all information.

Table 4. Trends in ERP components extracted for information processing for teeth

\begin{tabular}{|c|c|c|c|c|c|c|c|c|}
\hline \multicolumn{5}{|c|}{ Second year } & \multicolumn{4}{|c|}{ Fifth year } \\
\hline Latency & PC1 & $\mathrm{PC} 2$ & PC3 & ERP components & PC1 & PC2 & PC3 & PC4 \\
\hline $0-30$ & 0.292 & 0.141 & 0.549 & & -0.175 & -0.061 & 0.079 & -0.069 \\
\hline $31-60$ & 0.190 & 0.316 & 0.092 & N100 & 0.394 & 0.226 & 0.174 & -0.086 \\
\hline $61-90$ & 0.079 & 0.153 & 0.464 & & 0.089 & 0.237 & 0.811 & -0.116 \\
\hline $91-120$ & 0.228 & -0.084 & 0.868 & & 0.180 & -0.180 & 0.754 & 0.170 \\
\hline $121-150$ & 0.120 & 0.013 & 0.910 & IVIIVIN & 0.066 & 0.274 & 0.797 & 0.366 \\
\hline $151-180$ & 0.213 & 0.244 & 0.831 & & 0.238 & 0.215 & 0.594 & 0.580 \\
\hline $181-210$ & 0.591 & 0.207 & 0.520 & $\mathrm{~N} 2 \mathrm{~b}$ & 0.057 & 0.367 & 0.173 & 0.832 \\
\hline $211-240$ & 0.764 & 0.123 & 0.381 & & 0.500 & 0.268 & 0.103 & 0.747 \\
\hline $241-270$ & 0.853 & 0.112 & 0.263 & & 0.826 & 0.091 & 0.228 & 0.335 \\
\hline $271-300$ & 0.861 & 0.245 & 0.099 & & 0.898 & 0.092 & 0.215 & 0.063 \\
\hline $301-330$ & 0.831 & 0.269 & 0.063 & & 0.889 & 0.244 & 0.171 & -0.122 \\
\hline $331-360$ & 0.921 & 0.210 & 0.136 & P3a & 0.908 & 0.244 & 0.010 & 0.106 \\
\hline $361-390$ & 0.903 & 0.258 & 0.135 & & 0.913 & 0.255 & 0.112 & 0.128 \\
\hline $391-420$ & 0.872 & 0.349 & 0.133 & & 0.854 & 0.387 & 0.069 & 0.153 \\
\hline $421-450$ & 0.795 & 0.414 & 0.215 & & 0.665 & 0.576 & -0.054 & 0.236 \\
\hline $451-480$ & 0.794 & 0.454 & 0.183 & & 0.647 & 0.660 & -0.112 & 0.220 \\
\hline $481-510$ & 0.786 & 0.489 & 0.155 & & 0.599 & 0.703 & -0.140 & 0.204 \\
\hline $511-540$ & 0.703 & 0.574 & 0.098 & P3b & 0.543 & 0.727 & -0.157 & 0.287 \\
\hline $541-570$ & 0.649 & 0.670 & 0.062 & & 0.492 & 0.809 & -0.023 & 0.141 \\
\hline $571-600$ & 0.644 & 0.704 & 0.070 & & 0.409 & 0.805 & 0.138 & 0.208 \\
\hline $601-630$ & 0.478 & 0.829 & -0.028 & & 0.301 & 0.827 & 0.131 & 0.275 \\
\hline 631-660 & 0.091 & 0.828 & -0.160 & & -0.032 & 0.723 & 0.063 & 0.293 \\
\hline $661-690$ & 0.295 & 0.905 & 0.077 & SW & 0.295 & 0.872 & 0.222 & 0.183 \\
\hline 691-720 & 0.277 & 0.897 & 0.044 & & 0.187 & 0.928 & 0.161 & 0.087 \\
\hline $721-750$ & 0.222 & 0.875 & 0.128 & & 0.055 & 0.911 & 0.268 & -0.059 \\
\hline $\begin{array}{l}\text { C̄haracter- } \\
\text { value }\end{array}$ & 9.41 & 5.62 & 3.53 & & 7.35 & 7.32 & 2.69 & 2.46 \\
\hline $\begin{array}{l}\text { Cumlautive } \\
\text { proportion }\end{array}$ & 37.7 & 60.1 & 74.2 & & 29.4 & 58.7 & 69.4 & 79.2 \\
\hline
\end{tabular}

The value shows the factor loading. The PC shows a principal component. Characteristic value shows ratio that contributes to a principal component. Cumulative proportion shows ratio that each principal component accounts for in all information. 
P3a with a characteristic value of 7.35 ; the secondary principal component was a composite of $\mathrm{P} 3 \mathrm{~b}$ and SW, with a value of 7.32; the tertiary principal component was MMN, with a value of 2.69; and the quaternary principal component was $\mathrm{N} 2 \mathrm{~b}$, with a value of 2.46 . The cumulative proportion for the primary to tertiary principal components was $79.2 \%$.

\section{Discussion}

A tooth form is the sight information that has been sent by eyes is processed through a sight association area by a primary field of vision, and then tooth form is recognized. The concept of the tooth is collated with knowledge stored with a highly advanced association area and thinks following the general cognitive process that a meaning, and interpretation of the sight information is brought about afterwards.

The general subjective VAS scale was showed the tooth task was more difficult than the character in both second and fifth- year students. It is difficult to investigate how to recognize the tasks employing VAS or the correct answer rates by comparing the students from the two years. We cannot investigate differences in clinical experience knowledge. The reaction time for the character task compared to the tooth task was significantly shorter, leading to reduced decision-making times. However, no significant difference in clinical experience knowledge was noted between students from the two years. Reaction time corresponds to the combined time period of the stimulus processing system in the brain and reaction processing systems such as pushing the button after decision-making (18) and has conventionally been employed as a parameter in cognitive experiments (19). To recognize objects presented at different angles as in this experiment, mental rotation is involved in controlling representations of images in the brain. Concerning the mental rotation, Shepard \& Metzler (20) have reported that, when recognizing whether two graphics presented as stimuli at different angles would be the same, pattern cognition would involve turning an object imaged in the brain, which takes a certain period of time. In addition, reaction times do not directly represent information processing in the brain because they also include time taken by the reaction processing system involved in pushing the button. There are limitations with respect to investigations of pattern cognition including mental rotation based on reaction times.

Thus, at present, changes for such tasks in the neuroac- tive areas in the brain are identified by electroencephalographic analysis. An ERP is a brain potentials provoked by stimuli wherein a component of relative slow latency (100 $\mathrm{ms}$ or above) is known to reflect selective attention or cognitive performance and change. N100 is an involuntary potential associated with the transmission of a visual stimulus into the brain. $\mathrm{MMN}$ and $\mathrm{N} 2 \mathrm{~b}$ are related to matching processing between a pattern shape in the brain and a presented image. MMN is a simple pattern matching processing. $\mathrm{N} 2 \mathrm{~b}$ is reported to be potentially representative of a high-level pattern matching processing $(21,22)$; P3a representative of attention-orientation processing; $\mathrm{P} 3 \mathrm{~b}$ representative of working memory update processing (23-25); and SW to be representative of confirmation processing after information processing, as well as behavior performance processing, including preparation processing for the subsequent task (26). When we perform differentiation experiments, information processing varies depending on whether the task involves a part of the body. The commonly used hand task is considered to involve body schema such as motor control range, because an image is transformed to a subject's hand (27). Ebihara (3) a member of our class, has reported that the body schema may be involved in tooth differentiation employing ERP. Kuwahara and Ebihara (13) have studied information processing for teeth, characters, and hands employing the P300 and report no similarity between information processing for characters that do not involve body schema and the corresponding processing of tooth or hand. However, they were unable to investigate each information process extensively.

Donchin (28) has reported that ERP components can be objectively isolated and identified from average waveforms with slow potentials change through principal component analysis. This experiment applied principal component analysis of the average waveforms obtained with individual tasks and investigated trends in the occurrence of ERP components in differentiation-associated information processing. The principal component analysis extracted components that contribute more significantly than a principal component sequence. Moreover, it enables investigation of difficulty by observing trends in the extraction of individual principal components. The principal component analysis is used for the low dimension space without spoiling the information to have of multidimensional data as much as possible to separate from a wave form mixing information by the electroencephalographic analysis by a method to do 
an abridgement individually. The degree of difficulty of the task processing is examined by seeing a tendency to extraction of the principal component. Our study showed the following results: for the character task, the primary, secondary, tertiary, and quaternary principal components were a composite of N2b and P3a, P3b, SW, and MMN, respectively, in the second and fifth year students, indicating that the same principal components can be extracted in students from both years. This suggests that the same information processing was involved in discriminating characters in students from both years.

Information processing in the brain is reported to be rapid and efficient, without excessive loading on the cerebrum, and divided into automatic processing and attention processing for behavior decision-making $(29,30)$. In general, the former, in which input information is reconciled with images formed by knowledge and memory in the brain, is considered to precede the latter; if mismatching with images occurs, the information is subject to attention processing. Then it goes through decision processing for behavior decision, and then undergoes behavior performance processing. Since information processing for characters involves a higher percentage of pattern matching processing and attention-orientation processing for the primary principal component, we can assume that automatic and attention processing occurs in a similar manner. Decision-making is presumably less difficult because the character task involves differentiation of katakana, which requires common knowledge and simple shape recognition.

On the other hand, for the tooth task, the primary, secondary, and tertiary principal components were a composite of P3a and P3b, SW, and a composite of MMN and $\mathrm{N} 2 \mathrm{~b}$ in second-year students. The primary, secondary, tertiary, and quaternary principal components were P3a, a composite of P3b and SW, MMN, and N2b in fifth-year students. With respect to the primary and secondary principal components, these results indicate that the primary principal component of $\mathrm{P} 3 \mathrm{a}$ representing attentionorientation processing was isolated and identified in fifthyear students but not in second-year students. The secondary principal component, SW, representing behavior performance processing, was isolated and identified in second-year but not in fifth-year students. These results indicate that fifth-year students may differentiate tooth types based on a more effective decision-making process as "different" or "the same" than second-year students.
Furthermore, we extracted SW in second-year students, suggesting that they may take time to perform other associated tasks, such as confirmation, after decisionmaking.

In fifth-year students, the primary, tertiary, and quaternary principal components were clearly isolated as P3a, MMN, and N2b, respectively. This indicates that working memory controlled by the prefrontal area may perform at an early phase, an image of tooth may be judged to represent morphology in the temporal lobe without being subjected to mental rotation processing, and that the information processed may be projected to the frontal lobe of the cerebrum, leading to decision-making. Furthermore, possibility arresting you as a general viewpoint overlooking it that is not body intellect of a third person -like image namely the self and the body intellect of the partner has it pointed out because the superior temporal sulcus domain has inferior parietal lobule and communication as for the wave pattern of the neighborhood of $370 \mathrm{~ms}$ by Grriosman et al (31) so far . Therefore, it is thought that shortening of the reaction time does not need the time that mental rotation takes.

Based on these findings, students from both years evinced similar information processing for the character task, whereas each-year students evinced specific information processing for the tooth task. In particular, the results suggest the possibility that fifth-year students, who had acquired some clinical experience knowledge, may make their decisions based on a more effective cognitive approach involving working memory.

\section{Conclusion}

This study used event-related potentials to elucidate cognition processing among students who had acquired textbook-based dental knowledge and those who had also acquired clinical experience knowledge. The results indicate that student cohorts from both years may apply similar information processing approaches for the character task, whereas fifth-year students who had acquired experience knowledge may make their decisions based on more effective information processing for the tooth task.

\section{Acknowledgments}

The authors wish to express their deepest gratitude to Professors Takanori Ito and Satoshi Horihata, Nihon University School of Dentistry at Matsudo, for instructions 
throughout the study, as well as Sunao Iwaki Ph.D., from the Advanced Industrial Science and Technology (AIST) for his review of our work.

The authors also wish to thank individual members of the Department of Oral Diagnosis of the Nihon University School of Dentistry at Matsudo for their assistance with different aspects of this study.

\section{References}

1. Sackett, DL: Clinical diagnosis and clinical laboratory. Clin Invest Med, 1: 37-43, 1978.

2. Patel VL, Groen GJ, Frederiksen CH: Differences between medical students and doctors in memory for clinical cases. Med Educ, 20: 3-9, 1986.

3. Elstein AS, Schwarz A: Clinical problem solving and diagnostic decision making: selective review of the cognitive literature. BMJ, 324: 729-732, 2002.

4. Oyama K, Aoki S: The Role of anatomical components in tooth pattern cognition. Jpn J Oral Diag, 25: 113-120, 2012. (in Japanese)

5. Ebihara T: Changes in Event-related Potentials Associated with Mental Rotation. Jpn J Oral Diag, 26: 141-148, 2013. (in Japanese)

6. Kurosawa H, Aoki S, Ebihara T: Changes in event-related potentials related to pattern recognition of teeth in dental students. Int J Oaal-Med Sci, 13: 66-74, 2014.

7. Logan GD: Attention and preattention in theories of automaticity. Am J Psychol, 105: 317-339, 1992.

8. Treisman A, Vieira A, Hayes A: Automaticity and preattentive processing. Am J Psychol, 105: 341-362, 1992.

9. Ritter W, Vaughan HG Jr: Average evoked responses in vigilance and discrimination: a reassessment. Science, 164: 326-328, 1969.

10. Kutas M, McCarthy G, Donchin E: Augmenting mental chronometry: The P300 as a measure of stimulus evaluation time. Science, 197: 792-795, 1977.

11. Norman DA, Bobrow DG: On data-limited and resourcelimited processes.Cognitive Psychology, 7: 44-64, 1975.

12. Donchin E, Coles MGH: Is the P300 component a measure ofcontext updating? Behavioral and Brain Sciences. Science, 11: 357-374, 1988

13. Kuwahara K, Ebihara T: Event-related potentials related to pattern recognition of teeth based on body schema. Jpn J Oral Diag, 27: 133-141, 2014. (in Japanese)

14. Hillyard SA, Hink RF, Schwent VL, Picton TW: Electrical signs of selective attention in the human brain. Science, 182: 177-180, 1973.

15. Näätänen R, Paavilainen P, Rinne T, Alho K: The mismatch negativity (MMN) in basic research of central auditory processing. J Clin Neurophysiol. 118: 2544-2590, 2007.
16. Ullsperger PH, Gille G: The Late Positive Component of the ERP and Adaptation-Level Theory. Biol Psychol, 26: 299-306, 1988.

17. Squires NK, Squires KC, Hillyard SA: Two varieties of longlatency positive waves evoked by unpredictable auditory stimuli in man. Electroencephalogr Clin Neurophysiol, 38: 387-401, 1975

18. Adam N, Collins GI: Late components of the visual evoked potential to search in short-term memory. Electroencephalogr Clin Neurophysiol, 44: 147-156, 1978.

19. David E Meyer, Allen M Osman, David E Irwin, Steven Yantis: Modern mental chronometry. Biol Psychol, 26: 3-67, 1988.

20. Shepard RN, Metzler J: Mental rotation of three-dimentional objects. Science, 171: 701-703, 1971.

21. Näätänen R, Gaillard AWK, Mäntysalo S: Early selective attention effect on evoked potential reinterpreted. Acta Psychologica, 42: 313-329, 1978.

22. Logan GD: Attention and preattention in theories of automaticity. Am J Psychol, 105: 317-339, 1992.

23. Sutton S, Braren M, Zubin J, Join ER: Evoked-potential correlates of stimulus uncertainty. Science, 150: 1187-1188, 1965.

24. Halgren E, Marinkovic K, Chauvel P: Generators of the late cognitive potentials in auditory and visual oddball tasks. Electroencephalogr Clin Neurophysiol, 106: 156-164, 1998.

25. Knight RT, Scabini D: Anatomic bases of event-related potentials and their relationship to novelty detection in humans. J Clin Neurophysiol, 15: 3-13, 1998.

26. Aoki S: A cognitive science study on the knowledge acquisition of dental students as assessed at event-related potential. Jpn J Oral Diag, 11: 205-219, 1998.(in Japanese)

27. Amorim MA, Isableu B, Jarraya M: Embodied spatial transformation: "body analogy" for the mental rotation of Objects. J Exp Psychol Gen, 135: 327-347, 2006.

28. Donchin E: A multivariate approach to the analysis of average evoked potentials. IEEE Trans Biomed Eng, 13: 131-139, 1966.

29. Koyama S, Akahane-Yamada R, Gunji A, Kubo R, Roberts TPL, Yabe H, Kakigi R: Cortical evidence of the perceptual backward masking effect on $/ 1 /$ and $/ \mathrm{r} /$ sounds from a following vowel in Japanese speakers. Neuroimage, 18: 962-974, 2003.

30. Kasai K, Nakagome K, Itoh K, Koshida I, Hata A, Iwanami A, Fukuda M, Kato N: Impaired cortical network for preattentive detection of change in speech sound in schizophrenia: a high-resolution event-related potential study. Am J Psychiatry, 159: 546-553, 2002.

31. Grossman E, Donnelly M, Price R, Pickens D, Morgan V, Neighbor G, Blake R: Brain areas involved in perception of biological motion. J Cogn Neurosci, 12: 711-720, 2000. 